Isaac Gonçalves da Silva'

(1) https://orcid.org/0000-0003-1067-4765

Thatiana Araujo Maranhão²

o https://orcid.org/0000-0003-4003-1365

George Jó Bezerra Sousa ${ }^{3}$

O https:///orcid.org//0000-0003-0291-6613

Taynara Lais Silva'

(1) https:///rcid.org/0000-0003-1558-0506

Geovana Almeida dos Santos

Araujo ${ }^{1}$

O https://orcid.org/0000-0002-4088-6868

Daniele de Brito Sousa

Ohttps://orcid.org/0000-0001-5514-7235

Maria Lúcia Duarte Pereira ${ }^{4}$

OChtps://orcid.org/0000-0003-0079-5248

\section{Dinâmica temporal e espacial e fatores relacionados à mortalidade por suicídio entre idosos}

\author{
Temporal and spatial dynamics and factors related \\ to suicide mortality among the elderly
}

DOI: $10.1590 / 0047-2085000000367$

\section{RESUMO}

Objetivo: Analisar a dinâmica temporal e espacial e os fatores associados à mortalidade por suicídio entre idosos ( $\geq 60$ anos de idade) no Nordeste do Brasil. Métodos: Estudo ecológico que analisou os óbitos por suicídio ocorridos entre idosos na região Nordeste do Brasil, no período de 2010 a 2019. Foram realizadas análise temporal por Joinpoint, análise espacial segundo município de residência e análise multivariada pelo modelo Ordinary Least Squares Estimation, considerando-se $p<0,05$. Resultados: No período analisado, foi observado crescimento significativo de 3,0\% (IC95\%: 1,1-4,9; p < 0,001) ao ano na mortalidade por suicídio. Espacialmente, as taxas mais elevadas foram observadas prioritariamente em municípios do Piauí, Ceará e Rio Grande do Norte. Foram identificados quatro clusters de suicídio estatisticamente significativos ( $p<0,001)$. O cluster primário (2010-2019) abrangeu 141 municípios do Ceará, Rio Grande do Norte e Paraíba, que apresentaram risco 2,3 vezes maior de ocorrência de suicídio quando comparados aos demais municípios da região. Os indicadores índice de Gini $(\beta=14,02 ; p=$ $0,01)$, taxa de analfabetismo $(\beta=0,20 ; p<0,001)$ e taxa de envelhecimento $(\beta=0,36 ; p=0,02)$ apresentaram associação positiva com o suicídio entre idosos, enquanto razão de dependência $(\beta=-0,31$; $p<0,001)$ e taxa de desocupação $(\beta=-0,25 ; p<0,001)$ apresentaram associação negativa. Conclusão: Houve aumento significativo do suicídio entre idosos na região Nordeste, com maior concentração em quatro clusters espaciais localizados prioritariamente no Ceará, Paraíba, Piauí e Rio Grande do Norte. A associação com indicadores socioeconômicos reforça aspectos de vulnerabilidade dos idosos a esse tipo de agravo e auxilia na formulação de propostas de intervenções que promovam a sua redução.

\section{PALAVRAS-CHAVE}

Suicídio, idoso, epidemiologia, análise espacial, estudos de séries temporais.

\section{ABSTRACT}

Objective: To analyze the temporal and spatial dynamics and factors associated with suicide mortality among the elderly ( $\geq 60$ years of age) in Northeast of Brazil. Methods: This is an ecological study that analyzed deaths from suicide among elderly people in the Northeast region of Brazil, from 2010 to 2019. Temporal analysis by Joinpoint, spatial analysis according to municipality of residence and multivariate analysis by the Ordinary Least Squares Estimation model were performed, considering $p<0.05$. Results: During the analyzed period, a significant increase of 3.0\% (95\%Cl: 1.1-4.9; $\mathrm{p}<0.001)$ per year in suicide mortality was observed. Spatially, the highest rates were observed in municipalities of Piauí, Ceará and Rio Grande do Norte. Four statistically significant suicide clusters were identified ( $p<0.001$ ). The primary cluster (2010-2019) covered 141 municipalities in Ceará, Rio Grande do Norte and Paraíba, which presented a 2.3 times higher risk of suicide when compared to other municipalities in the region. The Gini index indicators $(\beta=14.02 ; p=0.01)$, illiteracy rate $(\beta=0.20 ; p<0.001)$ and aging rate $(\beta=0.36$; $p=0.02$ ) showed a positive association with suicide among the elderly, while the dependency ratio $(\beta=-0.31 ; p<0.001)$ and unemployment rate $(\beta=-0.25 ; p<0.001)$ had a negative association. Conclusion: There was a significant increase in suicide among the elderly in the Northeast region, with a greater concentration in four spatial clusters located primarily in Ceará, Paraíba, Piauí and Rio Grande do Norte. The association with socioeconomic indicators reinforces aspects of vulnerability of the elderly to this type of injury and helps in the formulation of intervention proposals that promote its reduction.

\section{KEYWORDS}

Suicide, elderly, epidemiology, spatial analysis, time series studies.

Received in: 13/Sep/2021. Approved in: 3/Dec/2021

1 Universidade Estadual do Piauí (UESPI), Parnaíba, PI, Brasil.

2 Cuidados Clínicos em Enfermagem e Saúde pela Universidade Estadual do Ceará (UECE), Fortaleza, CE, Brasil. Curso de Enfermagem da Universidade Estadual do Piauí (UESPI), Parnaíba, PI, Brasil.

3 Programa de Pós-graduação em Cuidados Clínicos em Enfermagem e Saúde da Universidade Estadual do Ceará (UECE), Fortaleza, CE, Brasil.

4 Universidade de São Paulo (USP), São Paulo, SP, Brasil. Programa de Pós-graduação em Cuidados Clínicos em Enfermagem e Saúde da Universidade Estadual do Ceará (UECE), Fortaleza, CE, Brasil.

Address for correspondence: Isaac Gonçalves da Silva. E-mail: isaacgslv@gmail.com 


\section{INTRODUÇÃO}

Nos últimos anos, tem-se observado rápido crescimento da população idosa brasileira, com aumento de 40,3\% no número de idosos no país, entre os anos de 2002 e 2012. No Brasil, é considerada idosa a pessoa que tem 60 anos ou mais de idade, e o crescimento da população idosa é cada vez mais expressivo em consequência da transição demográfica. Em 2010, existiam no país 20,5 milhões de idosos, o equivalente a aproximadamente 39 indivíduos para cada grupo de 100 jovens. Todavia, estima-se que em 2040 a população idosa represente $23,8 \%$ dos habitantes do país, transformando a proporção para quase 153 idosos para cada 100 jovens s., $^{1,2}$.

Entretanto, esse aumento tem sido acompanhado por uma tendência de crescimento das mortes autoprovocadas nesse grupo etário, as quais se distribuem de forma irregular devido às diferenças no contexto socioeconômico e cultural do país ${ }^{3}$. Nesse cenário, convém ressaltar que a idade se constitui em importante fator na determinação do suicídio, visto que as taxas de mortalidade por esse agravo tendem a ser mais elevadas entre pessoas idosas, em comparação com as demais faixas de idade, em quase todas as regiões do mundo ${ }^{4-6}$.

Estudos apontam várias situações que justificam taxas de suicídio tão expressivas na população idosa, tais como o declínio progressivo das reservas fisiológicas e da capacidade orgânica e funcional e o aparecimento de diversos tipos de doenças crônicas secundárias ao processo de envelhecimento ${ }^{7,8}$. Quando essas situações se somam à diminuição do autocuidado, à perda da autonomia e ao surgimento de crises existenciais, podem emergir percepções negativas para a pessoa idosa, as quais são determinantes na ocorrência de pensamentos suicidas e tentativas e concretização do ato de suicidar-se ${ }^{7,9,10}$.

No Brasil, embora as taxas de mortalidade por suicídio na população geral tenham apresentado aumento expressivo nos últimos anos, os óbitos ainda foram predominantes entre os indivíduos pertencentes à faixa etária de 60 anos ou mais $^{11,12}$. Na região Nordeste, a dinâmica observada não foi diferente, pois, em estudo que analisou os padrões de suicídio na população geral brasileira no período de 1996 a 2015, foi constatado aumento de 35,0\% nas taxas de mortes autoprovocadas entre idosos ${ }^{11}$.

Contudo, ressaltamos que o suicídio é um fenômeno que pode ser evitado ${ }^{4}$, o que torna ainda mais relevante a realização de investigações voltadas para o entendimento da ocorrência desse agravo na população idosa, sobretudo quando elas são conduzidas em nível regional. Assim, torna-se possível conhecer os locais onde há aumento da ocorrência de mortes autoprovocadas e entender quais os principais fatores relacionados ao problema.

Em face do exposto, o presente estudo se destaca pela utilização de ferramentas de geoprocessamento e emprego de análise de regressão multivariada, as quais permitem reconhecer os municípios que possuem riscos mais elevados para a ocorrência do suicídio dentro do período analisado e reconhecer os indicadores socioeconômicos que estiveram significativamente associados ao fenômeno para, dessa forma, auxiliar na formulação de propostas de intervenção que potencializem sua prevenção.

Portanto, diante da evidência do aumento da mortalidade autoprovocada na população idosa e do crescimento da ocorrência desse agravo na região Nordeste nos últimos anos, o presente estudo tem por objetivo analisar a dinâmica temporal e espacial e os fatores associados à mortalidade por suicídio entre idosos ( $\geq 60$ anos de idade) no Nordeste do Brasil, no período de 2010 a 2019.

\section{MÉTODOS}

Trata-se de um estudo ecológico, que analisou os óbitos por suicídio ocorridos entre indivíduos com 60 anos ou mais residentes na região Nordeste do Brasil, no período de 2010 a 2019. A região Nordeste tem uma população de 53.081 .950 pessoas, o que corresponde a cerca de $28,0 \%$ da população residente no Brasil, e extensão territorial de, aproximadamente, $1.554 .291 \mathrm{~km}^{2}$. Apresenta 1.794 municípios distribuídos por nove estados: Alagoas (AL), Bahia (BA), Ceará (CE), Maranhão (MA), Paraíba (PB), Pernambuco (PE), Piauí (PI), Rio Grande do Norte (RN) e Sergipe (SE) ${ }^{13}$.

Os dados foram obtidos por meio do Sistema de Informação sobre Mortalidade (SIM), do Departamento de Informática do Sistema Único de Saúde (Datasus) ${ }^{14}$, e se referem às mortes autoprovocadas entre idosos e que fazem menção ao suicídio na causa básica das declarações de óbito, sendo selecionados apenas os óbitos cujos códigos X60 ao X84 foram citados de acordo com a 10a Classificação Internacional de Doenças.

Para o cálculo das taxas de mortalidade brutas por suicídio, foi utilizado o software TabWin v.4.14. Como numerador da fórmula, foi utilizado o número de óbitos por suicídio entre idosos ocorridos em cada ano e, como denominador, a população de idosos nordestinos em cada ano considerado, multiplicado por 100.000 habitantes. Os dados brutos do suicídio em cada ano foram tabulados em planilha Excel e importados para o software Joinpoint Regression Program. A variação percentual anual (VPA) foi calculada com intervalo de confiança de 95\% (IC95\%), utilizando-se nível de significância de 5\% para testar a hipótese nula de que a VPA da série era igual a zero.

Desse modo, um valor negativo de VPA indicaria tendência decrescente e um valor positivo indicaria tendência crescente da mortalidade, enquanto valores de VPA não significantes representaram tendência de mortalidade estacionária. Cada ponto de inflexão adicionado ao modelo 
representa uma variação de tendência linear. Para tanto, ajustou-se o modelo assumindo-se que o número de pontos de inflexão poderia variar de zero até dois no decorrer dos anos. Foi definido como variável independente o ano da ocorrência do suicídio e como variável dependente a taxa de mortalidade em cada ano, calculadas e padronizadas diretamente no programa ${ }^{15}$.

Para a análise estatística espacial, foi utilizada base cartográfica digital com arquivo vetorial do tipo shapefile, obtida no sítio eletrônico do Instituto Brasileiro de Geografia e Estatística (IBGE). A base contém polígonos que delimitam as fronteiras políticas dos estados e municípios do Nordeste brasileiro, tendo sido georreferenciada na projeção Universal Transversa de Mercator (UTM), zonas 22 a 25 Sul, datum WGS 84 (World Geodetic System 1984). Os dados tabulares com informações demográficas e sobre a mortalidade foram ligados à tabela dos objetos da camada geográfica utilizando-se geocódigos padronizados pelo IBGE comuns aos dois arquivos.

Inicialmente, foi criado o mapa temático das taxas brutas de suicídio entre idosos para cada município da região analisada. Em seguida, com o objetivo de minimizar a instabilidade das taxas brutas, foi realizada a suavização dessas taxas por meio do método Bayesiano Empírico Local. Nesse estudo, a estimativa Bayesiana Empírica Local utilizou somente os vizinhos geográficos da área na qual se estimara a taxa, sendo, portanto, menos instáveis, pois levam em consideração no seu cálculo não só a informação da área, como também a informação da sua vizinhança.

A estrutura de dependência entre os valores observados do suicídio entre idosos nos municípios nordestinos foi analisada por meio da autocorrelação espacial. Foi aplicado o Índice de Moran Global para testar a hipótese de dependência espacial e fornecer uma medida geral de associação para toda a área do estudo. Após a constatação de presença de autocorrelação espacial global, o Índice de Moran Local (Local Index Spatial Analysis - LISA) verificou a presença de agregados espaciais e quantificou o grau de associação espacial em cada município do conjunto amostral, considerando os agregados estatisticamente significantes com nível de significância de 5\%.

Os resultados do Índice de Moran Local estão representados pelo Moran Map e pelo Lisa Map. Na análise dos padrões espaciais, evidenciados no Moran Map, foi possível observar quatro tipos: Alto/Alto, que representa municípios com altas taxas de suicídio circundados por municípios com taxas igualmente altas; Baixo/Baixo, que representa municípios com baixas taxas de suicídio com vizinhos também com baixas taxas, assim como Alto/Baixo e Baixo/Alto, que demonstram áreas de transição epidemiológica, cujo padrão espacial pode variar ao longo do tempo ${ }^{16}$.

A formação de aglomerados puramente espaciais de óbitos por suicídio foi avaliada por meio da técnica estatística de varredura Scan puramente espacial. Conjuntamente, foram gerados mapas espaciais do risco relativo (RR) para formação de aglomerados de óbitos na região no período 2010-2019. Foram utilizados o modelo probabilístico de Poisson e uma janela geográfica de base circular que englobou $5 \%$ da população sob risco, com nível de significância de 5\%. Quanto à análise do RR para a formação de aglomerados no período estudado, este representa a intensidade da ocorrência dos óbitos por suicídio em uma determinada área, quando comparada às demais áreas da região analisada. Onde se verifica $R R>1$, o RR de uma área específica é superior ao risco da totalidade da região.

O software TerraView v.4.2.2 foi utilizado para a obtenção da matriz de vizinhança e para o cálculo da estatística bayesiana e das estimativas globais e locais de autocorrelação espacial. Para a análise de varredura, foi utilizado o software SaTScan v.9.6 e, para a construção dos mapas temáticos, foi empregado o software QGis v.2.14.17.

Para compor a construção do modelo de regressão linear multivariada Ordinary Least Squares Estimation (OLS), foram coletados 10 indicadores socioeconômicos referentes à população de cada município nordestino no Portal do Atlas do Desenvolvimento Humano no Brasil ${ }^{17}$. Os indicadores inseridos no modelo foram: índice de desenvolvimento humano municipal; índice de Gini; taxa de envelhecimento; taxa de analfabetismo - 18 anos ou mais de idade; taxa de desocupação - 18 anos ou mais de idade; esperança de vida ao nascer; razão de dependência; percentual de 18 anos ou mais de idade com ensino fundamental completo; percentual de vulneráveis à pobreza e renda per capita.

Para o modelo multivariado, foram inseridas as taxas de mortalidade e as informações socioeconômicas de todos os 1.794 municípios da região Nordeste, utilizando-se o método backward e critério epidemiológico. A presença de colinearidade entre as variáveis independentes foi verificada por meio do Variance Inflation Factor (VIF). As variáveis não colineares foram inseridas no modelo OLS, adotando-se significância estatística de 5\%. Para o cálculo do modelo multivariado OLS, foi utilizado o software Stata v. $12^{\circ}$.

Este estudo foi aprovado pelo Comitê de Ética em Pesquisa da Universidade Estadual do Piauí sob parecer no 3.286.816/2019 e Certificado de Apresentação de Apreciação Ética de no 07557818.4.0000.5209. Ressaltase que todos os preceitos éticos e legais foram respeitados conforme preconizado pelas Resoluções no 466/12 e n॰ 510/2016 do Conselho Nacional de Saúde.

\section{RESULTADOS}

Na região Nordeste do Brasil, no período de 2010 a 2019, foram registrados 4.368 óbitos por suicídio entre os indivíduos com 60 anos ou mais. A taxa média de mortalidade no período analisado foi de 6,9 óbitos por 100 mil habitantes. 
As mortes autoprovocadas foram predominantes entre os indivíduos do sexo masculino ( $n=3.527 ; 80,8 \%$ ), com faixa etária de 60 a 69 anos $(n=2.279 ; 52,2 \%)$, escolaridade de um a sete anos de estudo $(n=1.733 ; 52,6 \%)$, casados $(n=2.136$; $53,8 \%)$ e de cor/raça parda $(n=2.935 ; 71,5 \%)$. A maioria dos óbitos ocorreu no domicílio da vítima ( $n=2.929 ; 67,3 \%)$, sendo o enforcamento, estrangulamento e sufocação o método mais escolhido para cometer o suicídio ( $n=3.141 ; 71,9 \%)$ (dados não mostrados em tabelas).

Foi possível observar que, no período de 2010 a 2019, houve crescimento estatisticamente significativo $(p<0,001)$ de 3,0\% (IC95\%: 1,1-4,9) ao ano no suicídio entre os idosos nordestinos. Quando analisado segundo o sexo, também foi observado crescimento significativo $(p<0,001)$ entre os idosos do sexo masculino de 2,9\% (IC95\%: 1,1-4,8) ao ano (Tabela 1).

Entre os estados da região Nordeste, houve variação de tendência com a inserção de um ponto de inflexão apenas no estado de Pernambuco, visto que no período de 20102017 o estado apresentou crescimento significativo ( $p<$ 0,001) de 7,2\% (IC95\%: 4,5-10,0) ao ano no suicídio entre idosos, entretanto no período 2017-2019 observou-se estabilidade com tendência estacionária. Também foram observadas tendências crescentes e significativas $(p<0,001)$ na mortalidade por suicídio entre idosos nos estados da Bahia (VPA: 4,4; IC95\%: 1,2-7,8), Maranhão (VPA: 6,6; IC95\%: 3,0-10,2) e Piauí (VPA: 5,3\%; IC95\%: 1,7-9,0) (Tabela 1).

Na figura 1 A podemos observar que as taxas brutas de suicídio estão distribuídas de forma irregular por toda a região Nordeste, com a maioria dos municípios apresentando taxas de até 35,33 óbitos por 100 mil habitantes. Após aplicado o método Bayesiano Empírico Local, nota-se uma distribuição mais uniforme, com um padrão espacial mais evidente. As taxas bayesianas mais elevadas se concentraram especialmente em parcela expressiva dos municípios do Piauí, Ceará, Rio Grande do Norte e Sergipe, com taxas iguais ou superiores a 10,0 óbitos por 100.000 habitantes (Figura 1B).

O índice de Moran Global demonstrou a presença de autocorrelação espacial positiva $(I=0,15 ; p=0,001)$. Quanto aos aglomerados de suicídio entre idosos obtidos por meio do gráfico de espalhamento de Moran, foi possível observar, nos municípios em vermelho, padrão Alto/Alto, localizado predominantemente no Piauí, assim como em alguns municípios do Ceará, Rio Grande do Norte e Paraíba. Já as áreas em verde indicam os municípios com padrão espacial Baixo/ Baixo, observado principalmente na região extremo oeste e sul da Bahia, no norte do Maranhão e no leste de Alagoas, fazendo fronteira com parte da Zona da Mata pernambucana (Figura 1C).

É importante ressaltar que o Moran Map confirmou os resultados da estatística bayesiana quanto à localização dos municípios nos quais o problema da mortalidade por suicídio na população idosa se mostra mais grave. Todos os municípios que apresentaram um padrão espacial no Moran Map (Figura 1C) se mostraram significantes no Lisa Map (Figura 1D), com $p<0,05$.

A figura $1 \mathrm{E}$ aponta os 10 clusters espaciais identificados por meio do método de varredura Scan, contudo apenas quatro deles apresentaram significância estatística $(p<0,001)$. O cluster tido como primário (em vermelho), isto é, aquele que possui a menor probabilidade de ter ocorrido ao acaso, incluiu 141 municípios, estando eles predominantemente

Tabela 1. Variação percentual anual da mortalidade por suicídio entre idosos ( $\geq 60$ anos de idade) na região Nordeste, Brasil, 2010-2019

\begin{tabular}{|c|c|c|c|c|}
\hline Variáveis & Periodo & $\begin{array}{l}\text { Variação percentual anual } \\
\text { (IC } 95 \%^{\star} \text { ) }\end{array}$ & Valor de $\mathbf{p}$ & Tendência \\
\hline \multicolumn{5}{|l|}{ Sexo } \\
\hline Ambos os sexos & 2010-2019 & $3,0(1,1-4,9)$ & $<0,001$ & Crescente \\
\hline Feminino & 2010-2019 & $3,4(-0,2-7,1)$ & 0,10 & Estacionária \\
\hline Masculino & 2010-2019 & $2,9(1,1-4,8)$ & $<0,001$ & Crescente \\
\hline \multicolumn{5}{|l|}{ Estado } \\
\hline Alagoas & 2010-2019 & $-0,5(-7,6-7,2)$ & 0,90 & Estacionária \\
\hline Bahia & 2010-2019 & $4,4(1,2-7,8)$ & $<0,001$ & Crescente \\
\hline Ceará & 2010-2019 & $1,3(-1,1-3,7)$ & 0,20 & Estacionária \\
\hline Maranhão & 2010-2019 & $6,6(3,0-10,2)$ & $<0,001$ & Crescente \\
\hline Paraíba & 2010-2019 & $2,6(-1,0-6,4)$ & 0,10 & Estacionária \\
\hline \multirow[t]{2}{*}{ Pernambuco } & $2010-2017$ & $7,2(4,5-10,0)$ & $<0,001$ & Crescente \\
\hline & 2017-2019 & $-13,9(-27,3-2,0)$ & 0,10 & Estacionária \\
\hline Piauí & 2010-2019 & $5,3(1,7-9,0)$ & $<0,001$ & Crescente \\
\hline Rio Grande do Norte & 2010-2019 & $0,5(-2,8-3,9)$ & 0,70 & Estacionária \\
\hline Sergipe & 2010-2019 & $-2,0(-5,8-2,0)$ & 0,30 & Estacionária \\
\hline
\end{tabular}

*IC95\%: índice de confiança de 95\%. 
nos estados do Ceará, Rio Grande do Norte e Paraíba. Clusters secundários (em amarelo) foram observados principalmente nos municípios do Piauí, Ceará e Sergipe e, assim como o cluster primário, também possuem importância epidemiológica, uma vez que também apresentaram significância estatística ( $p<0,001)$. Dentre aqueles com RR mais elevado, destacaram-se os municípios de Belém do Piauí (PI) e Monte das Gameleiras (RN), os quais possuem risco para o suicídio 15,31 e 17,94 vezes maior que o risco observado em toda a região Nordeste, respectivamente (Figura 1F).
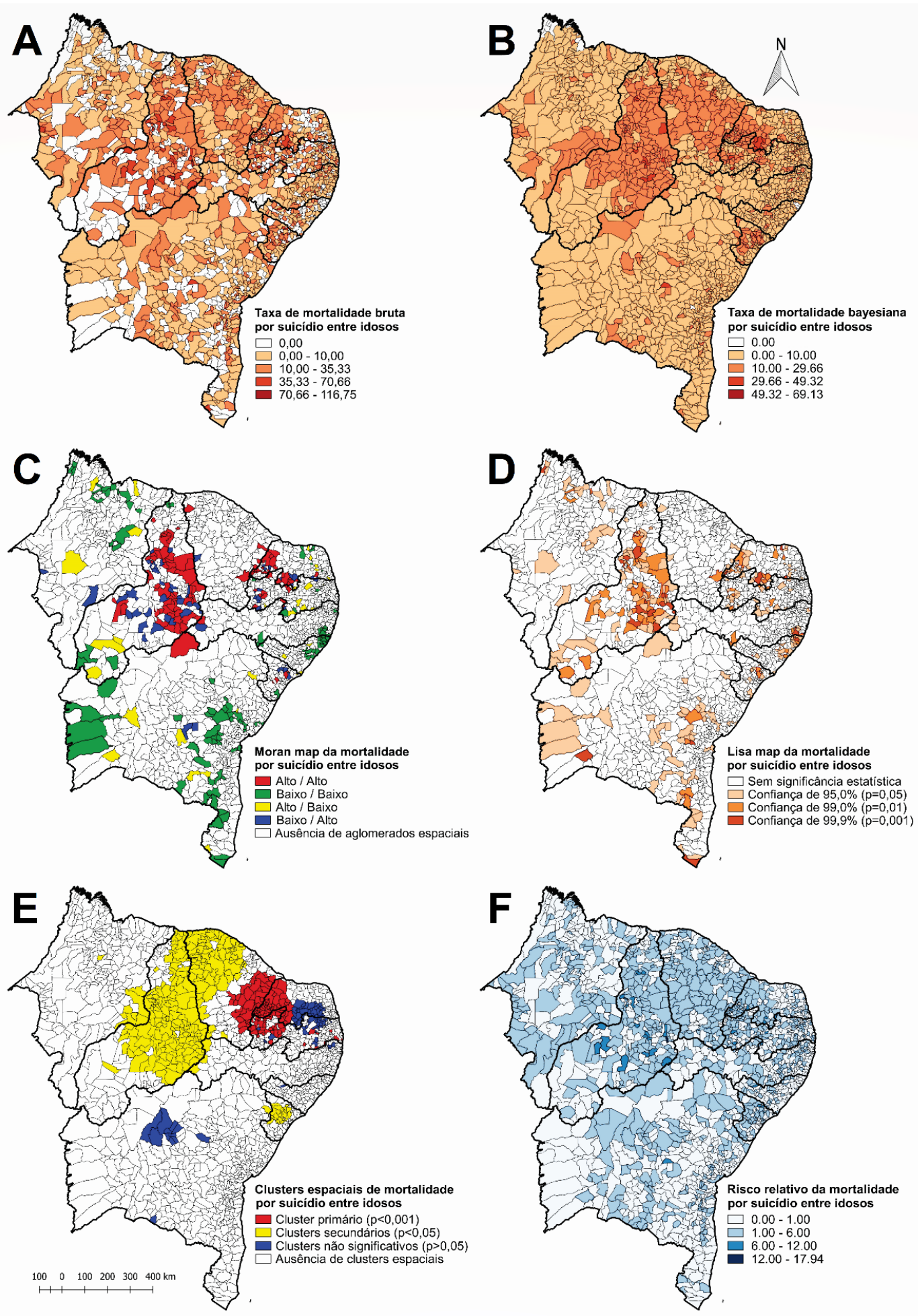

Figura 1. Distribuição da taxa de mortalidade bruta $(\mathbf{A})$ e bayesiana $(\mathbf{B})$ do suicídio entre idosos, Moran Map (C), Lisa Map (D), clusters espaciais (E) e risco relativo (F) do suicídio entre idosos ( $\geq 60$ anos de idade) na região Nordeste, Brasil, 2010-2019. 
A tabela 2 traz informações detalhadas dos aglomerados de óbitos por suicídio identificados por meio do método de varredura Scan puramente espacial. O cluster primário possui raio de 141,91km e os municípios que o compõem têm, em média, 2,30 vezes mais risco de suicídio, em comparação aos demais municípios da região estudada.

Os resultados da análise de regressão multivariada OLS revelaram associação positiva entre o suicídio na população idosa e os indicadores socioeconômicos índice de Gini ( $\beta=$ $14,02 ; p=0,01)$, taxa de analfabetismo $(\beta=0,20 ; p<0,001)$ e taxa de envelhecimento $(\beta=0,36 ; p=0,02)$. Em contrapartida, os indicadores razão de dependência $(\beta=-0,31 ; p<$ $0,001)$ e taxa de desocupação $(\beta=-0,25 ; p<0,001)$ apresentaram associação negativa com a mortalidade por suicídio entre idosos. Nesse modelo, o coeficiente de determinação $\left(R^{2}\right)$ foi de 0,0417 , o que significa dizer que o conjunto dos indicadores socioeconômicos selecionados foi capaz de explicar parcialmente a variabilidade do suicídio em 4,17\% (Tabela 3).

\section{DISCUSSÃO}

No presente estudo, foi evidenciado crescimento significativo da mortalidade autoprovocada entre os idosos residentes na região Nordeste. Esse resultado está em concordância com investigação que analisou as mortes por suicídio entre idosos no Brasil, em que foi observada tendência de crescimento significativo da mortalidade no período de 2000 a 2014, com maior incremento entre os indivíduos do sexo masculino ${ }^{3}$.

Entre os estados nordestinos, Bahia, Maranhão, Pernambuco e Piauí apresentaram os maiores aumentos anuais nas taxas de suicídio. Relacionado a isso, estudo que analisou a tendência temporal do suicídio nos estados brasileiros, no período de 1997 a 2015, também observou tendência de crescimento das mortes autoprovocadas entre indivíduos com 60 anos ou mais nos estados do Piauí, Maranhão e Bahia. Além disso, foi observado ainda que o agravo apresentou tendência de crescimento entre os

Tabela 2. Aglomerados espaciais dos óbitos por suicídio entre idosos ( $\geq 60$ anos de idade) na região Nordeste, Brasil, 2010-2019

\begin{tabular}{|c|c|c|c|c|c|c|c|c|}
\hline Cluster & $\mathrm{N}^{\circ}$ de municípios & Estados* & Raio (km) & $\mathrm{N}^{0}$ de casos & $\begin{array}{l}N^{0} \text { esperado de } \\
\text { casos }\end{array}$ & $\mathbf{R R}^{\star \star}$ & $\operatorname{LLR}^{\star \star \star}$ & Valor de $p$ \\
\hline 1 & 141 & $\mathrm{CE}, \mathrm{PB}, \mathrm{PE}$ & 141,91 & 458 & 211,02 & 2,30 & 115,42 & $<0,001$ \\
\hline 2 & 151 & CE, MA, PE, PI & 214,71 & 323 & 141,37 & 2,38 & 89,21 & $<0,001$ \\
\hline 3 & 105 & $\mathrm{CE}, \mathrm{PI}$ & 181,61 & 331 & 216,78 & 1,57 & 27,45 & $<0,001$ \\
\hline 4 & 30 & BA, SE & 54,48 & 77 & 37,22 & 2,08 & 16,37 & $<0,001$ \\
\hline 5 & 67 & $\mathrm{~PB}, \mathrm{RN}$ & 76,14 & 102 & 64,98 & 1,58 & 9,12 & 0,120 \\
\hline 6 & 1 & BA & 0,00 & 9 & 2,38 & 3,78 & 5,35 & 0,964 \\
\hline 7 & 11 & BA & 76,22 & 39 & 22,04 & 1,77 & 5,32 & 0,968 \\
\hline 8 & 1 & $\mathrm{AL}$ & 0,00 & 5 & 0,80 & 6,23 & 4,95 & 0,997 \\
\hline 9 & 3 & PE & 11,44 & 15 & 6,06 & 2,47 & 4,65 & 0,999 \\
\hline 10 & 1 & BA & 0,00 & 7 & 1,69 & 4,13 & 4,62 & 0,999 \\
\hline
\end{tabular}

*AL: Alagoas; BA: Bahia; CE: Ceará; MA: Maranhão; PB: Paraíba; PE: Pernambuco; PI: Piaúi; RN: Rio Grande do Norte; SE: Sergipe.

${ }^{*}$ RR: risco relativo para o cluster comparado ao resto do estado.

***LLR: teste da razão de verossimilhança logarítmica.

Tabela 3. Indicadores associados a mortalidade por suicídio entre idosos ( $\geq 60$ anos de idade) na região Nordeste, Brasil, 2010-2019

\begin{tabular}{|c|c|c|c|c|}
\hline Indicadores socioeconômicos* & Coeficiente $(\beta)$ & IC 95\%** & 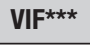 & Valor de $p$ \\
\hline Índice de Gini & 14,02 & $(03,37-24,67)$ & 1,16 & 0,01 \\
\hline Razão de dependência & $-0,31$ & $(-0,40--0,21)$ & 1,64 & $<0,001$ \\
\hline Taxa de analfabetismo (18 anos ou mais) & 0,20 & $(0,12-0,28)$ & 1,57 & $<0,001$ \\
\hline Taxa de desocupação (18 anos ou mais) & $-0,25$ & $(-0,38--0,11)$ & 1,15 & $<0,001$ \\
\hline Taxa de envelhecimento & 0,36 & $(0,07-0,65)$ & 1,24 & 0,02 \\
\hline
\end{tabular}

${ }^{*}$ Coeficiente de determinação $\left(R^{2}\right)=0,0417$.

**IC 95\%: índice de confiança de 95\%.

***VIF: Variance Inflation Factor. 
idosos do sexo masculino em todos os estados nordestinos, excetuando-se o estado de Pernambuco, onde a tendência se manteve estável ${ }^{12}$.

Nessa perspectiva, o aumento das mortes autoprovocadas nas populações idosas tem se tornado um relevante problema de saúde pública nos últimos anos, principalmente por se tratar de mortes evitáveis e que, muitas vezes, são negligenciadas ${ }^{4}$. Investigações apontam que, para cada óbito autoprovocado na velhice, há a ocorrência de ideações e tentativas suicidas em pelo menos outros quatro indivíduos nessa faixa etária, o que pode justificar possíveis aumentos na tendência de suicídio nessa população ${ }^{8}$.

Alguns fatores, como a maior fragilidade ocasionada pelo próprio processo de envelhecimento, maior vulnerabilidade ao surgimento de crises existenciais, sobretudo às relacionadas aos transtornos mentais, além da maior frequência de problemas de saúde física e incapacidades, isolamento social e baixo apoio familiar, podem interferir diretamente na saúde mental da pessoa idosa e aumentar as chances de comportamentos suicidas ${ }^{18,19}$.

$\mathrm{Na}$ análise estatística espacial, foi observado que municípios dos estados do Piauí, Ceará, Rio Grande do Norte e Paraíba apresentaram altas taxas de mortalidade nos resultados da estimativa bayesiana empírica local. Outra investigação que analisou os padrões espaciais do suicídio no Brasil, no período de 1990 a 2015, observou, no ano de 2014, a presença de um cluster na região do Semiárido Nordestino, que abrangeu os estados do Piauí, Ceará, Rio Grande do Norte, Paraíba e Pernambuco, evidenciando que a população dos municípios que faziam parte desse aglomerado possuía risco 1,39 vezes maior de cometer o suicídio ${ }^{11}$.

Desse modo, é reconhecido que o suicídio é influenciado por fatores sociais e econômicos, e o aumento da mortalidade por esse fenômeno entre os idosos nordestinos pode ainda receber influências dos indicadores socioeconômicos desfavoráveis que se fazem presentes em parcela relevante dos municípios dessa região. Nesse contexto, fatores como desigualdade social, baixa renda e desemprego podem interferir na qualidade de vida da maioria das famílias e induzir a ocorrência do suicídio 3,20.

Todavia, não se descarta a possibilidade de que esse aumento dos casos de suicídio no Nordeste seja consequência também dos possíveis avanços de cobertura do SIM, como resultado da melhoria da captação dos dados e alimentação do sistema, embora ainda seja elevado o número de casos preenchidos de forma incorreta atribuindo-se outras causas de morte e/ou com campos respondidos como ignorados ou não informados ${ }^{21}$.

A análise de regressão multivariada verificou associação positiva entre a ocorrência do suicídio e a variável independente índice de Gini, de modo que à medida que o valor do índice aumenta, também é elevada a ocorrência do suicídio. Considerando que o índice de Gini é um importante indicador que avalia a desigualdade, é possível inferir que quanto maior é a desigualdade de renda, mais elevadas serão as taxas de suicídio ${ }^{22}$. Nesse contexto, estudo que analisou o impacto dos fatores socioeconômicos sobre a mortalidade por suicídio na Colômbia observou que a maior desigualdade de renda eleva o risco de suicídio em quase três vezes ${ }^{23}$.

Além disso, foi verificado que a taxa de analfabetismo esteve associada positivamente ao suicídio entre idosos. O aumento da desigualdade social, que está frequentemente associada à baixa escolaridade, ao desemprego e à pobreza, interfere diretamente no modo de vida do indivíduo e nas suas relações sociais, pois aumenta as chances de condições de vida precárias e insatisfatórias e diminui o grau de satisfação pessoal, o que constitui condições importantes para levar uma pessoa a pensar em suicídio 5,24,25.

Foi observado ainda que à medida em que a taxa de envelhecimento aumenta, a mortalidade por suicídio entre idosos também se eleva na mesma proporção. Diante do exposto, reflete-se que o aumento do número de pessoas com idade avançada pode aumentar o risco de suicídio nessa faixa etária, tanto pelas situações de risco e eventos estressantes que a velhice traz à tona como devido a morte de pessoas queridas, surgimento de doenças e comprometimento cognitivo ${ }^{10,26}$. Além disso, na velhice se tornam mais comuns a diminuição das atividades de lazer, o aumento da solidão, isolamento social e sentimentos de inutilidade e peso para a família do idoso 7,27.

A variável razão de dependência mostrou-se negativamente associada ao suicídio, o que corrobora estudo realizado no Rio Grande do Norte, que também observou essa relação inversa. O autor enfatiza a maior ocorrência do agravo na população economicamente ativa antes da aposentadoria ${ }^{22}$. Todavia, é importante ressaltar que o aumento no grau de dependência gera inúmeros conflitos no relacionamento entre idosos e seus familiares e cuidadores, principalmente entre o público masculino, que sente a necessidade de perpetuar sua posição de autoridade como chefe da família e provedor. Assim, muitos deles não aceitam a dependência de alguém para realizar suas necessidades da vida diária, gerando tensões e resistência em contribuir nessa nova fase de sua vida ${ }^{28}$.

Também foi observada associação negativa entre a variável taxa de desocupação e o suicídio. Desse modo, quando se analisa que a região Nordeste possui sua principal fonte de renda nos setores agrícolas, com parte considerável dos trabalhadores rurais utilizando mão de obra familiar, observa-se a possibilidade de transtornos mentais e pensamentos suicidas ocasionados pelas fragilidades laborais vivenciadas. Tais problemas são ocasionados pelas condições de trabaIho precárias, jornadas de trabalho intensas e cansativas, clima desfavorável e baixa produtividade, frequentemente se necessitando de complementação de renda com outras atividades 20,29 . 
Entre as limitações do presente estudo, podem ser citados o uso de dados secundários oriundos de um sistema de informações que está sujeito a inconsistências na qualidade de suas informações devido ao preenchimento de dados incorretos. Diante disso, faz-se necessária maior atenção durante a coleta de dados e alimentação do sistema, com preenchimento completo das informações solicitadas, de modo a diminuir casos ignorados, os quais não possuem peso em análises de estudos epidemiológicos. Ademais, ressalta-se a possibilidade de subnotificações em consequência da classificação incorreta da causa do óbito. Todavia, essas limitações não inviabilizaram a condução do estudo, tampouco reduziram sua magnitude.

\section{CONCLUSÕES}

No período de 2010 a 2019, foi evidenciado crescimento estatisticamente significativo da mortalidade por suicídio entre os idosos da região Nordeste, especialmente entre homens. A análise espacial revelou taxas mais elevadas de mortes autoprovocadas nos municípios dos estados do Ceará, Paraíba, Piauí, Rio Grande do Norte e Sergipe, sendo identificados quatro clusters espaciais significativos, localizados prioritariamente nos municípios do Ceará, Paraíba, Piauí e Rio Grande do Norte. Foi encontrada associação positiva entre a mortalidade por suicídio na população idosa e os indicadores socioeconômicos índice de Gini, taxa de analfabetismo e taxa de envelhecimento, enquanto os indicadores razão de dependência e taxa de desocupação apresentaram associação negativa com a mortalidade por suicídio entre idosos.

Tais achados contribuem para a formulação de propostas de intervenção que visem à redução da ocorrência do suicídio entre idosos, fornecendo evidências que auxiliam na implementação e reformulação de políticas públicas de saúde mental e entendimento de como o fenômeno se distribui na região analisada e os principais fatores relacionados.

\section{CONTRIBUIÇÕES INDIVIDUAIS}

Isaac Gonçalves da Silva e Thatiana Araujo Maranhão Contribuíram significativamente na concepção e delineamento do estudo, coleta, análise e interpretação dos dados e redação do artigo.

George Jó Bezerra Sousa - Contribuiu significativamente para a análise estatística e interpretação dos dados.

Taynara Lais Silva, Geovana Almeida dos Santos Araujo e Daniele de Brito Sousa - Contribuíram substancialmente na interpretação dos dados e na redação do artigo.

Thatiana Araujo Maranhão, George Jó Bezerra Sousa e Maria Lúcia Duarte Pereira - Contribuíram significativamente na revisão crítica e relevante do conteúdo intelectual.
Todos os autores contribuíram para a aprovação final da versão a ser publicada.

\section{CONFLITOS DE INTERESSES}

Os autores declaram não possuir conflitos de interesse.

\section{REFERÊNCIAS}

1. Ministério da Saúde (BR). Saúde da pessoa idosa [Internet]. 2020. Disponível em: https:// www.gov.br/saude/pt-br/assuntos/saude-de-a-a-z-1/s/saude-da-pessoa-idosa. Acesso em: 18 mar. 2021.

2. Miranda GMD, Mendes ACG, Silva ALA. Population aging in Brazil: current and future social challenges and consequences. Rev Bras Geriatr Gerontol. 2016;19(3):507-19.

3. Santos EGO, Oliveira YOMC, Azevedo UN, Nunes ADS, Amador AE, Barbosa IR. Spatial temporal analysis of mortality by suicide among the elderly in Brazil. Rev Bras Geriatr Gerontol. 2017;20(6):845-55.

4. Brasil. Ministério da Saúde. Secretaria de Vigilância em Saúde. Tentativas e suicídios na população idosa do Brasil. Boletim Epidemiológico. v. 51. Brasilia: Ministério da Saúde; 2020.

5. Bachmann S. Epidemiology of suicide and the psychiatric perspective. Int J Environ Res Public Health. 2018;15(7):1-23.

6. Li LW, Xu H, Zhang Z, Liud J. An ecological study of social fragmentation, socioeconomicdeprivation, and suicide in rural China: 2008-2010. SSM - Population Health. 2016;2:365-72.

7. Oliveira JMB, Vera I, Lucchese R, Silva GC, Tomé EM, Elias RA. Aging, mental health, and suicide. An integrative review. Rev Bras Geriatr Gerontol. 2018;21(4):488-98.

8. Marques VSNF, Sousa MNA, Nogueira RBSS, Nogueira TBSS, Oliveira IC. Suicídio em idosos brasileiros: retrato de uma realidade. Rev Bras Multidiscip. 2020;23(3):190-202.

9. Adinkrah M. Characteristics of Elderly Suicides in Ghana. Omega (Westport). 2018;82(1):3-24.

10. Hernandez SC, Overholser JC, Philips KL, Lavacot J, Stockmeier CA. Suicide among older adults: Interactions among key risk factors. Int J Psychiatry Med. 2021;56(6):408-21.

11. Palma DCA, Santos ES, Ignotti E. Análise dos padrões espaciais e caracterização dos suicídios no Brasil entre 1990 e 2015. Cad Saude Publica. 2020;36(4):e00092819.

12. Rodrigues CD, Souza DS, Rodrigues HM, Konstantyner TCRO. Trends in suicide rates in Brazil from 1997 to 2015. Braz J Psychiatry. 2019;41(5):380-8.

13. Instituto Brasileiro de Geografia e Estatística (IBGE). Censo demográfico 2010 [Internet]. 2010. Disponível em: https://censo2010.ibge.gov.br/. Acesso em: 2 fev. 2021.

14. Ministério da Saúde (BR). Departamento de Informática do Sistema Único de Saúde (Datasus). Informações de Saúde (Tabnet) - Datasus [Internet]. 2020. Disponível em: https://datasus.saude.gov.br/informacoes-de-saude-tabnet/. Acesso em: 2 abr. 2021.

15. Sousa GJB, Garces TS, Pereira MLD, Moreira TMM, Silveira GM. Temporal pattern of tuberculosis cure, mortality, and treatment abandonment in Brazilian capitals. Rev Lat Am Enfermagem. 2019;27:e3218.

16. Silva BFA, Prates AAP, Cardoso AA, Rosas N. 0 suicídio no Brasil contemporâneo. Soc Estado. 2018;33(2):565-79.

17. Programa das Nações Unidas para o Desenvolvimento (PNUD). Atlas do Desenvolvimento Humano no Brasil [Internet]. 2021. Disponível em: http://www.atlasbrasil.org.br/. Acesso em: 4 jul. 2021

18. Minayo MCS, Figueiredo AEB, Mangas RMN. Estudo das publicações científicas (20022017) sobre ideação suicida, tentativas de suicídio e autonegligência de idosos internados em Instituiçōes de Longa Permanência. Ciênc Saúde Colet. 2019;24(4):1393-404.

19. Altın̈ZZ AE, Yenilmez Ç, Öner SKD, Yldız P. Completed suicide rates of older adults in 5-year age bands in Turkey between 2002 and 2013: A retrospective study. Geriatr Gerontol Int. 2018;19(1):66-9.

20. Santos EG0, Barbosa IR. Conglomerados espaciais da mortalidade por suicídio no Nordeste do Brasil e sua relação com indicadores socioeconômicos. Cad Saúde Coletiva. 2017;25(3):371-8. 
21. Carmo ÉA, Santos PHS, Ribeiro BS, Soares (J, Santana MLADA, Bomfim EDS, et al. Características sociodemográficas e série temporal da mortalidade por suicídio em idosos no estado da Bahia, 1996-2013. Epidemiol Serv Saúde. 2018;27(1):e20171971.

22. Amaral SS. Suicídio no RN e sua relação com determinantes espaciais, urbanização, desenvolvimento e outros fatores socioeconômicos. Rev Bras Estud Reg Urbanos. 2019;13(2):288-308

23. Dávila-Cervantes (A, Pardo-Montaño AM. Impacto de factores socioeconómicos en la mortalidad por suicidios en Colombia, 2000-2013. Gerenc Políticas Salud. 2017;16(33):36-51.

24. Choi JW, Kim TH, Shin J, Han E. Poverty and suicide risk in older adults: A retrospective longitudinal cohort study. Int J Geriatr Psychiatry. 2019;34:1565-71.

25. Kim JW, Jung HY, Won DY, Shin YS, Noh JH, Kang TI. Landscape of elderly suicide in South Korea: Its trend according to age, gender, and educational attainment. Omega (Westport). 2020;82(2):214-29.
26. Koo YW, Kölves K, De Leo D. Suicide in older adults: differences between the young-old, middle-old, and oldest old. Int Psychogeriatr. 2017;29(8):1297-306.

27. Costa ALS, Souza MLP. Narrativas de familiares sobre o suicídio de idosos em uma metrópole amazônica. Rev Saude Publica. 2017;51:121.

28. Bruinsma JL, Beuter $M$, Borges $Z N$, Jacobi $C$ da $S$, Benetti ERR, Venturini $L$, et al. Conflitos interpessoais de idosos: revisão integrativa de literatura. Res Soc Dev. 2020;9(12):e43391211146.

29. Leite JF, Dimenstein M, Macedo JPS, Dantas CB, Silva EL, Sousa AP de. Lifestyle, Mental Health and Gender Conditions in Rural Contexts: A Study within Agraria Reform Settlements of Northeastern Brazil. Av Psicol Latinoam. 2017;35(2):301. 